\title{
Controlled Release of Proteins Bound to Spherical Polyelectrolyte Brushes
}

\author{
By A. Wittemann, B. Haupt, and M. Ballauff* \\ Physikalische Chemie I, University of Bayreuth, D-95440 Bayreuth, Germany
}

Polyelectrolyte Brushes / Protein / Adsorption / Albumin / Counterions

\begin{abstract}
We discuss the interaction of proteins dissolved in aqueous solution with spherical polyelectrolyte brushes (SPB). The SPB consist of a solid core particle of colloidal dimensions ( $c a .100 \mathrm{~nm}$ in diameter) onto which long polyelectrolyte chains have been grafted. Immersed in aqueous solution of proteins these SPB will take up high amounts of protein if the ionic strength is low. At high ionic strength, however, virtually no protein will enter into the brush layer attached to the surface of the core particles. We show that bovine serum albumin (BSA) bound at low ionic strength will gradually be released upon raising the salt concentration in the solution in a well-controlled manner: For each raise of the ionic strength in solution there is a well-defined amount of protein that is released. We show that BSA adsorbed to a conventional carboxylated latex will not be released if treated in the same manner. All findings, namely the uptake of protein as well as the controlled release can be explained by the "counterion release force": Patches of positive charge on the surface of the proteins which are immersed in the brush layer become multivalent counterions of the polyelectrolyte chains thus releasing a concomitant number of counter- and coions. Release of counterions as induced by the adsorption of proteins is hence the main driving force for the polyelectrolyte-mediated protein adsorption (PMPA).
\end{abstract}

\section{Introduction}

The interaction of proteins with solid surfaces is an important question that has a tremendous technical implication [1,2]. Very often adsorption of proteins is to be avoided to prevent fouling as e.g. in medical applications. On the other hand, modern biotechnology requires often the immobilization of proteins and enzymes for applications in processing and diagnostics [3]. The technical importance thus delineated has led to an enormous literature that is hard to overlook (see Refs. [1,2,4] for further citations). A comprehensive un-

* Corresponding author. E-mail: Matthias.Ballauff@uni-bayreuth.de 
Fig. 1. Scheme of the radial structure of the spherical polyelectrolyte brushes used in this study. Attachment of chains of the weak polyelectrolyte poly(acrylic acid) leads to an annealed brush $[13,14]$. The $\mathrm{pH}$ in the system is chosen to be high enough in order to ensure full ionization of the charged groups within the brush layer [13]. The average distance $D$ between the chains on the surface is of the order of a few nanometers only. Hence, the polyelectrolyte chains strongly interact and form a polyelectrolyte brush [1214]. The thickness $L$ of the brush layer can be tuned by the salt concentration $c_{\mathrm{a}}$ in the system that determines the ionic strength $c_{\mathrm{S}}$ within the brush layer through a Donnanequilibrium.

derstanding is therefore necessary in order to predict whether a given protein will adsorb on a surface or not.

Polymer chains attached to solid surfaces [5] are often used to prevent the adsorption of proteins from the aqueous phase [1]. The most important example of a protein-resistant coating is a dense layer of poly(ethyleneoxide) grafted to the surface [5-8]. The repulsive interaction of such a layer with proteins may first of all be explained by steric interaction [5]: Embedding a protein in a dense layer of polymer chains is followed by a loss of conformational entropy of the system [6-8]. Steric repulsion between dense grafted polymer layers and dissolved colloidal objects as e.g. proteins must hence be a major factor when considering possible mechanisms for preventing protein adsorption.

If the polymer chains attached to the solid surface are carrying the same charge as the dissolved proteins, a strong repulsion is expected to result from both steric interaction and electrostatic repulsion. However, we have demonstrated that bovine serum albumin (BSA) and several other proteins adsorb strongly on colloidal particles that carry long polyelectrolyte chains $[9,10]$. Figure 1 shows schematically the radial structure of these particles [11-13]. 
Long chains of poly(acrylic acid) (PAA) or poly(styrene sulfonic acid) (PSS) are grafted densely to poly(styrene) latex particles that are dispersed in water. These particles have been synthesized by a grafting-from technique [11, 12] and characterized extensively by dynamic light scattering $[12,13]$. The linear dimensions of the grafted chains are of the order of 50 to $250 \mathrm{~nm}$ whereas the distance between two chains directly at the surface is of the order of 3-6nm only. Hence, the dense layer of linear chains is in the so-called brush limit and the particles are termed spherical polyelectrolyte brushes (SPB) [14]. The main feature of the polyelectrolyte brush is the strong localization of the counterions within the brush layer $[15,16]$. Hence, the concentration $c_{\mathrm{s}}$ of the co- and counterions within the brush may deviate from the concentration $c_{\mathrm{a}}$ in the system (see Fig. 1). In this way the brush layer creates a local environment that can differ widely from the surrounding liquid.

Recently, we found that BSA as well as other proteins strongly adsorb onto the SPB in aqueous solution if the ionic strength is low whereas no adsorption takes place at high concentrations of added salt [9, 17-20]. Hence, the adsorption of proteins takes place on the "wrong side" of the isoelectric point $\mathrm{pI}$, that is, for a $\mathrm{pH}>\mathrm{pI}$ so that the proteins are carrying more negative than positive charges. The steric and electrostatic repulsion operating between negatively charged brushes and dissolved proteins carrying negative charges as well is obviously overcompensated by another force that leads to strong adsorption.

Previous studies have shown that i) the secondary structure of the adsorbed BSA, $\beta$-lactoglobulin and of ribonuclease A is nearly fully preserved [17, 19], ii) the proteins are evenly distributed within the brush layer [21,22], and iii) that the enzyme activity of adsorbed enzymes as e.g. glucoamylase is largely preserved $[18,20]$. The same conclusion was drawn from a study of the fluorescence activity of the fluorescent protein mEosFP [23]. Moreover, strong adsorption is also seen for planar polyelectrolyte brushes [24, 25]. It has hence become evident that the "polyelectrolyte-mediated protein adsorption" (PMPA) onto spherical polyelectrolyte brushes is a general phenomenon.

In this paper we discuss for the first time the reverse process in a quantitative fashion: We show that an adsorbed protein, namely BSA, can be released in a controlled manner by a gradual raise of the ionic strength. In order to compare this behavior to the adsorption on conventional surfaces, we shall present data on the adsorption of BSA to a carboxylated latex [1,2]. The controlled release of proteins adsorbed onto SPB is discussed in terms of the "counterion release force" [25-29] introduced earlier [9]: Adsorbed proteins can become multivalent counterions of the polyelectrolyte chains in the brush layer thus releasing a concomitant number of counter- and coions. The release experiments to be discussed here will be shown to be in full accord with this explanation. 


\section{Experimental}

The SPB were prepared and characterized as described recently $[9,12]$. The SPB were generated by affixing PAA-chains onto the surface. The SPBparticles used here termed $\mathrm{KpS13}$ are characterized as follows [9]: core radius $R=51 \mathrm{~nm}$, contour length $L_{\mathrm{c}}=36 \mathrm{~nm}$, and the grafting density $\sigma=0.13 \mathrm{~nm}^{-2}$. The hydrodynamic radius $R_{H}$ was determined by dynamic light scattering in dilute solution as described in previous work $[9,12,13]$.

The synthesis of the carboxylated poly(styrene) latex was carried out by emulsion polymerization along the lines of the synthesis of the poly(styrene) core of the SPB [11, 12]. $5 \mathrm{Mol} \%$ of acrylic acid were added to the batch. Appropriate amounts of surfactant and initiator were chosen to obtain particles with a radius of $55 \mathrm{~nm}$ which is comparable to the core radius of the SPB. All systems have been purified carefully by extensive ultrafiltration.

All aqueous solutions of the SPB were carefully adjusted to a given ionic strength and $\mathrm{pH}$. As in previous work we used N-morpholinoethanesulfonic acid (MES) adjusted by $\mathrm{NaOH}$ to a $\mathrm{pH}$ of 6.1 [9]. All investigations were done at a MES-concentration of $10 \mathrm{mM}$. Higher ionic strengths were adjusted through addition of $\mathrm{NaCl}$ to these solutions. In order to avoid possible microbial growth, $2 \mathrm{mM} \mathrm{NaN}_{3}$ were added to all solutions [9].

Bovine serum albumin (BSA, fatty acids free, Sigma A-6003) was purchased from Sigma and used without further purification. Solutions of BSA were prepared in buffer solutions (MES) with defined concentrations of added salt. These solutions were added to the SPB in buffer solution (MES) to obtain $1 \mathrm{wt}$ \% SPB in the final solution. The suspensions were equilibrated for 24 hours. Thereafter the solutions were filled into a serum-replacement cell and flushed eight times with buffer solution of same $\mathrm{pH}$ and salinity. The amount of protein washed off by this procedure was determined spectroscopically from the extinction at $278 \mathrm{~nm}$ (absorption coefficient $\epsilon_{278}=44.300 \mathrm{M}^{-1} \mathrm{~cm}^{-1}$. Subsequently, this figure and the total amount was used to calculate the amount of adsorbed BSA.

The adsorption of BSA onto the carboxylated latex was done exactly under the same conditions. Solutions containing various amounts of BSA were added to suspensions of the latex to obtain a $1 \mathrm{wt}$ \% latex in the resulting solution. The amount of unbound protein was flushed away after equilibrating the solutions for 24 hours and determined spectroscopically.

Desorption experiments were done as follows: $5 \mathrm{~mL}$ of a suspension of SPB (weight fraction of the SPB: 0.02) onto which $948 \mathrm{mg}$ BSA per gram of particles had been adsorbed previously were placed in an ultrafiltration cell. Ultrafiltration was done by flushing this solution by $180 \mathrm{~mL}$ of buffer solution (10 mM MES buffer) to which various amounts of sodium chloride had been added. Figure 2 shows this experiment in a schematic manner. The total amount of desorbed protein was determined spectroscopically. 


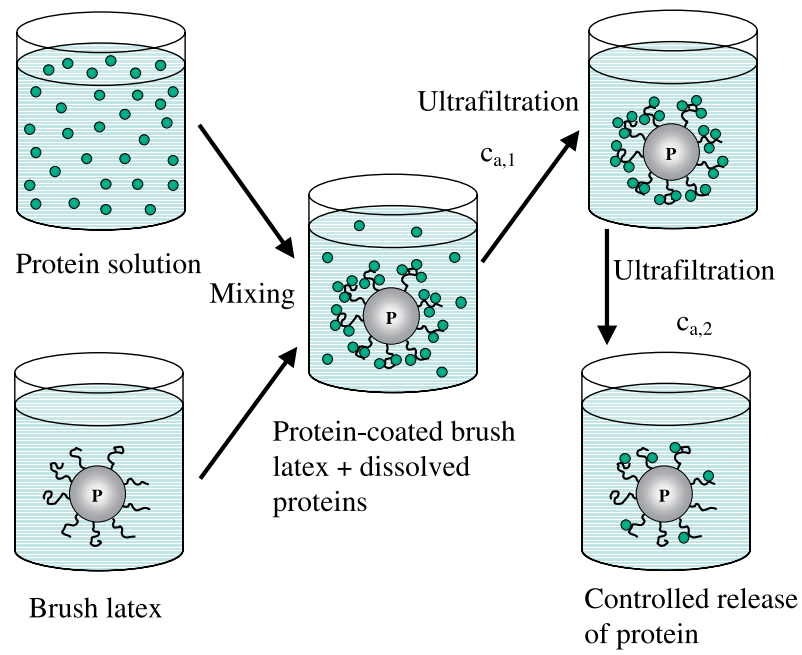

Fig. 2. Schematic representation of the experiment: Solutions of BSA were prepared in buffer solutions with defined concentrations of added salt. These solutions were added to the SPB dissolved in the same buffer. After equilibration for 24 hours the non-adsorbed protein was removed by careful serum replacement [9]. For the desorption experiment BSA is first adsorbed onto the spherical polyelectrolyte brushes with a defined concentration of added salt of low ionic strength $c_{\mathrm{a}, 1}$. Unbound protein is flushed away by ultrafiltration against buffer solution of the same ionic strength. The controlled release of the BSA is induced by ultrafiltration with buffer solution of higher ionic strength $c_{\mathrm{a}, 2}$.

The same experiment was done using the carboxylated latex onto which $140 \mathrm{mg}$ of BSA had been adsorbed previously. Ultrafiltration was done by flushing this solution by $180 \mathrm{~mL}$ of buffer solution ( $10 \mathrm{mM}$ MES buffer) to which various amounts of sodium chloride had been added. The desorbed protein was determined spectroscopically.

\section{Results and discussion}

\subsection{Adsorption curves}

We first discuss the adsorption of proteins onto the spherical polyelectrolyte brushes. As for the model protein we use BSA as in previous experiments [9]. The filled circles in Fig. 3 give the resulting adsorption curve. In order to compare this to the well-studied case of the adsorption of proteins on unmodified surfaces, we shall present the adsorption isotherm of BSA onto a carboxylated latex (filled squares in Fig. 3). The carboxylated latex consists of poly(styrene) particles that bear carboxyl-groups on their surface. The radius is $55 \mathrm{~nm}$ and these particles can thus directly be compared to the core particles of the SPB. The marked difference between the two results is immediately obvious: The 


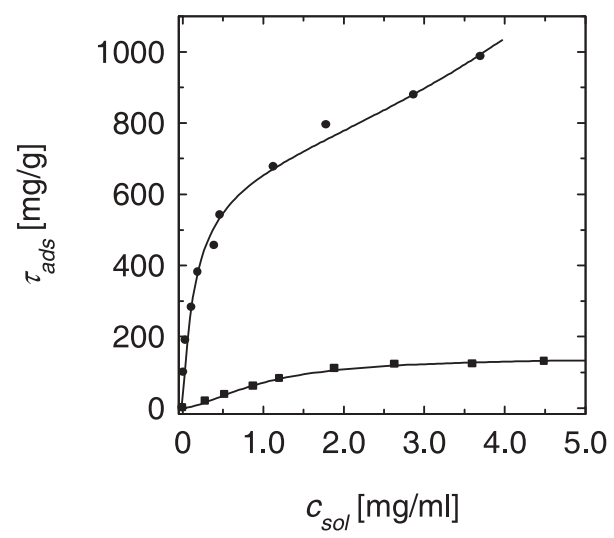

Fig. 3. BSA adsorption on SPB (circles) and on conventional carboxylated poly(styrene) particles (squares) above the isoelectric point (5.1). The amount of adsorbed protein $\tau_{\text {ads }}$ per unit mass particles is plotted against the concentration of the protein left in solution $c_{\text {sol }}$. The data points are fitted by Eq. (1). The $\mathrm{pH}$ is 6.1 and the ionic strength is $7 \mathrm{mM}$. See text for further explanation.

adsorption isotherm of BSA onto a conventional carboxylated latex levels off soon resulting in a monolayer of protein on the surface. Hence, if full coverage of the surface is reached, no further protein will be adsorbed under these conditions. Much higher amounts of proteins are adsorbed onto the SPB and the maximum values of $\tau_{\text {ads }}$ widely exceeds the value expected for a monolayer.

As shown previously $[9,20]$, the adsorption can be described by giving the amount of adsorbed protein per gram of the SPB $\tau_{\text {ads }}$ as the function of the concentration $c_{\text {sol }}$ of the protein remaining in solution. In this way the resulting curves resemble much the usual adsorption isotherms. All data of $\tau_{\text {ads }}$ as the function of $c_{\text {sol }}$ obtained so far $[9,17,20]$ can be described by the following expression that was derived from the conventional BET-isotherm treating the adsorption of multiple layers $[9,20]$ :

$$
\frac{\tau_{\mathrm{ads}}}{\tau_{\mathrm{ads}, \mathrm{M}}}=\frac{z w_{\mathrm{ads}} c_{\mathrm{sol}}^{1 / n}}{\left(1-w_{\mathrm{ads}} c_{\mathrm{sol}}^{1 / n}\right)\left[1+(z-1) w_{\mathrm{ads}} c_{\mathrm{sol}}^{1 / n}\right]} .
$$

We consider two fractions: One fraction of protein molecules enters deeply and shows thus a higher adsorption energy and a second fraction which is loosely bound in the periphery of the brush. $\tau_{\mathrm{ads}, \mathrm{M}}$ denotes the maximum amount of strongly bound protein. The probability for the adsorption of this part of the adsorbed protein is given by $z w_{\text {ads }} c_{\text {sol }}$ with $z>1$. The adsorption energy of the loosely bound protein is much lower and can be averaged. The probability of the adsorption of the less strongly bound protein is given by $w_{\text {ads }} c_{\text {sol }} ; n$ is an empirical parameter which takes into account the fact that 
the adsorption energy may depend on the degree of adsorption. Hence, in this model the adsorption can be described in terms of four adjustable parameters $\tau_{\text {ads, },}, w_{\text {ads }}, z, n[20]$.

In case of the carboxylated latex the curves of $\tau_{\text {ads }}$ as the function of $c_{\text {sol }}$ correspond to a Langmuir-like adsorption with a maximum adsorption $\tau_{\text {ads, } M}$ of $140 \mathrm{mg}$ BSA per g particles. Given the Stokes diameter of BSA of $7 \mathrm{~nm}$ and its molecular mass $(66.300 \mathrm{~g} / \mathrm{Mol})$, this adsorption degree refers to complete coverage of the surface with a BSA monolayer.

For BSA adsorbed onto the SPB we obtain a $\tau_{\text {ads, } M}$ of $680 \mathrm{mg}$ BSA per $\mathrm{g}$ particles. SAXS experiments demonstrated that the protein molecules enter deeply in the brush and are more or less equally distributed within the brush [21]. Hence, the protein molecules are not adsorbed onto a solid surface as considered by the BET-theory. Please note that $\tau_{\text {ads, M }}$ may thus refer to several layers of protein within the brush. Compared to the carboxylated latex, the fivefold higher amount of bound BSA has to be attributed to multiple layers of protein embedded within the polyelectrolyte brush.

\subsection{Controlled release of adsorbed proteins}

All experimental investigations done so far demonstrate that the polyelectrolyte-mediated protein adsorption is a spontaneous process [9, 10, 17-20]. Figure 2 shows the experiment in a schematic fashion: The protein and the spherical polyelectrolyte brush (SPB) particles are mixed in an aqueous buffer solution of defined ionic strength $c_{\mathrm{a}, 1}$. The PMPA process takes place and the unbound protein is removed by ultrafiltration against a buffer solution of the same ionic strength. It needs to be noted that the ultrafiltration in this step is done with a large excess of solution. Yet, no protein is liberated. This experimental finding points to a non-equilibrium state within the brush layer. If there would be an equilibrium distribution of the protein between the SPB and the solution, the process of ultrafiltration would flush away all the bound protein as well. This, however, is not observed.

The reverse process, that is the controlled release of the adsorbed protein BSA is done by ultrafiltration in the same way as the adsorption experiment (see Fig. 2): BSA is first adsorbed strongly to the SPB at low ionic strength $c_{\mathrm{a}, 1}(0.007 \mathrm{M})$, and unbound protein is flushed away. The release of proteins is induced by flushing this solution with a given amount of a solution having a higher ionic strength $c_{\mathrm{a}, 2}$. The amount of protein released in this step is measured. In this way the amount of released protein is obtained as the function of the concentration $c_{\mathrm{a}, 2}$.

Figure 4 displays the percentage of the amount of BSA released at a given $c_{\mathrm{a}, 2}$. No protein is released at low ionic strength. However, increasing of $c_{\mathrm{a}}$ leads to the controlled release of more and more protein. The highest amount of protein is released between 0.06 and $0.1 \mathrm{M}$. There is only a remainder of 10 to $20 \%$ of the protein that is kept firmly within the brush layer. This experiment 
Fig. 4. Desorption of the protein BSA bound on the SPB (filled circles) and on the carboxylated latex (triangles) upon raising the ionic strength in the system. BSA is first adsorbed onto the SPB and on the carboxylated latex at low ionic strength $(0.007 \mathrm{M})$. The fraction of desorbed BSA obtained for both systems is plotted versus the concentration $c_{\mathrm{a}}$ of added salt. The open circles give the ionic strength $c_{\mathrm{S}}$ within the brush layer of the SPB (see Eq. (3)). The dashed line marks $c_{\mathrm{S}}=c_{\mathrm{a}}$. See Fig. 2 and text for further explanation.

demonstrates that raising $c_{\mathrm{a}}$ within the solution leads to a new, well-defined state in which the amount of adsorbed protein is lower.

These experimental findings are in strong contrast to the results of the release experiments done on conventional carboxylated latexes. The triangles in Fig. 4 display the amounts of released BSA for different ionic strength. Virtually no BSA is liberated under these conditions. The absence of any release is not that surprising of course. One should rather expect an increase in the binding strength as electrostatic repulsions among the protein molecules and to the like-charged support are screened by addition of salt (see Ref. [2] and further literature given there). Moreover, it has been shown that adsorption of proteins onto such surfaces leads to denaturation [30,31]. Hence, the comparison shown in Fig. 4 demonstrates clearly that the process of adsorption onto these two different systems must have different reasons which will be discussed in the following:

Both the adsorption experiment as well as the controlled release upon raising $c_{\mathrm{a}}$ show that the main driving force must be related to the ionic strength $c_{\mathrm{S}}$ within the brush layer [32]. This can be demonstrated by calculating $c_{\mathrm{S}}$ within the brush layer as the function of $c_{\mathrm{a}}$. Following Russel and coworkers [32-34] the brush layer can be treated in terms of a classical Donnan-equilibrium [12, 13,35]. The brush layer is characterized by its thickness $L$ ( $c f$. Fig. 1). The concentration of the counterions $c_{\mathrm{ci}}$ is given by the number of charges within the brush layer because we assume full dissociation. Counterion condensation $[36,37]$ is taken into account by assuming that counterions will condense 
onto the polyelectrolyte chains until the distance between two charges is given by the Bjerrum-length $l_{\mathrm{B}}\left(l_{\mathrm{B}}=0.714 \mathrm{~nm}\right.$ in water at $\left.25^{\circ} \mathrm{C}\right)$ [32]. Therefore concentration of osmotically active counterions $c_{\mathrm{ci}}$ follows as (cf. Ref. [32])

$$
c_{\mathrm{ci}}=\frac{3 R^{2} \sigma L_{\mathrm{c}}}{l_{\mathrm{B}}\left[(R+L)^{3}-R^{3}\right] N_{\mathrm{A}}},
$$

where $R$ is the radius of the core particles, $\sigma$ is the number of grafted chains per unit area, $L_{\mathrm{c}}$ is the contour length of the grafted chains, $N_{\mathrm{A}}$ is Avogadro's number, and $\sigma$ is the number of chains per unit area ( $c f$. Fig. 1). The thickness $L$ is determined for each concentration of added salt $c_{\mathrm{a}}$ by dynamic light scattering $[9,12,13]$. Given the value of $c_{\mathrm{ci}}$, the ionic strength $c_{\mathrm{S}}$ within the layer including the counterions follows from the Donnan-equilibrium as $[13,32]$

$$
c_{\mathrm{S}}=c_{\mathrm{a}}\left(1+\left(\frac{c_{\mathrm{ci}}}{2 c_{\mathrm{a}}}\right)^{2}\right)^{1 / 2} .
$$

The open circles in Fig. 4 display the ionic strength $c_{\mathrm{S}}$ thus calculated. As already discussed previously, $c_{\mathrm{S}} \gg c_{\mathrm{a}}$ for low $c_{\mathrm{a}}$. This reflects the localization of the counterions within the brush layer. For higher and higher $c_{\mathrm{a}}$ the concentration inside and outside the brush layer approach each other.

Figure 4 demonstrates that the release of BSA is obviously related to the raise of $c_{\mathrm{S}}$ within the brush to values of the order of $0.1 \mathrm{M}$. The comparison shown in Fig. 4 hence gives the proof that the controlled release of the protein from the brush layer is directly related to the ionic strength $c_{\mathrm{S}}$ within the layer. In particular, a marked step-like release of BSA occurs at $c_{\mathrm{a}} \approx c_{\mathrm{S}}$. There is only a remainder of protein that cannot be washed out even for the highest ionic strength. This points to other interactions of the protein with the polyelectrolyte brush as e.g. hydrophobic attraction $[1,2]$ that is not related to $c_{\mathrm{a}}$. In opposite to this, the adsorption of BSA onto a carboxylated latex is fully determined by hydrophobic attraction and no liberation can take place at all.

\subsection{Polyelectrolyte-mediated protein adsorption: driving forces}

Based on these results we shall discuss the driving forces of the PMPA as well as the reverse process, namely the controlled release of the bound proteins in the following. Two different models have been discussed in literature so far: i) A charge reversal of the protein by a $\mathrm{pH}$ lower within the brush layer than outside [38]; and ii) the counterion release forces [25-29]. Both effects have already been discussed previously in a qualitative manner [9].

\subsubsection{Charge reversal}

The localization of counterions within a weak polyelectrolyte is necessarily followed by a smaller $\mathrm{pH}$ within the brush layer if the ionic strength is low. 
This effect and its implication for the PMPA was recently discussed in detail by Biesheuvel and Wittemann [38]. By suitable combination of the $\mathrm{pH}$ adjusted in the system and if $c_{\mathrm{a}}$ is low, the local $\mathrm{pH}$ within the brush layer may be lower than the $\mathrm{pI}$ of the protein. Hence, the net charge of the protein is reversed and a strong electrostatic attraction between unlike charged objects becomes operative. The theory of Biesheuvel and Wittemann [38] predicts therefore a strong dependence on the $\mathrm{pH}$ and the adsorption is predicted to vanish for a sharply defined $\mathrm{pH}$.

Previous experiments, however, have revealed that the $\mathrm{pH}$ is a parameter of secondary importance [9]. This can be seen from the discussion of Fig. 8 of Ref. [9]. Here $\tau_{\text {ads }}$ is plotted against $c_{\text {sol }}$. Parameter is the $\mathrm{pH}$ which ranges from a value just above the isoelectric point $\mathrm{pI}$ up to a value more than two units above the isoelectric point. There is a very pronounced adsorption of BSA in the immediate vicinity of the isoelectric point. The curves of $\tau_{\text {ads }}$ as the function of $c_{\text {sol }}$ will therefore shoot up and only bend over at very high values of $\tau_{\text {ads }}$. In this region charge reversal is certainly operative and leads to a marked adsorption of proteins. However, strong adsorption still takes place at considerably higher $\mathrm{pH}$. This points to the fact that the $\mathrm{pH}$ is an important but not a decisive parameter.

A further argument can be derived from the adsorption curve of glucoamylase discussed in Ref. [20]. This enzyme has an isoelectric point of $\mathrm{pI}=3.5$ whereas the adsorption experiment was done at a $\mathrm{pH}$ of 6.1 . Hence, $\mathrm{pH}$ is markedly higher than $\mathrm{pI}$ and charge reversal cannot be invoked anymore in order to explain the strong interaction of the protein and the brush layer. It must be traced back to different reason discussed in the subsequent section.

\subsubsection{Counterion release}

As mentioned above, the counterions are mostly localized within the brush layer $[15,16]$. Equation 3 shows that in the limit of salt-free solutions the ionic strength $c_{S}$ within the brush is given by the concentration $c_{\mathrm{ci}}$ of the counterions. This leads to an enormous osmotic pressure for salt-free systems ("osmotic brush") which in turn will stretch the polyelectrolyte chains of the brush layer to nearly full length [13,14,22,35,39-41]. This is quite in opposite to what is found for linear polyelectrolytes in solution. Here only the Manning fraction of the counterions is immobilized by the electric field of the linear macroion $[36,37]$. For typical polyelectrolytes as e.g. the poly(acrylic acid) used for the synthesis of the SPB, this fraction is of the order of $75 \%$, that is, osmotic coefficient giving the fraction of free counterions is around 0.25 [37].

Hence, we suggest here that the strong osmotic pressure inside the brush layer is one of the driving forces for the PMPA. This can be argued in the following way: Proteins bear positive and negative charges on their surface. A part of these charges may be grouped together in "patches" containing a few 


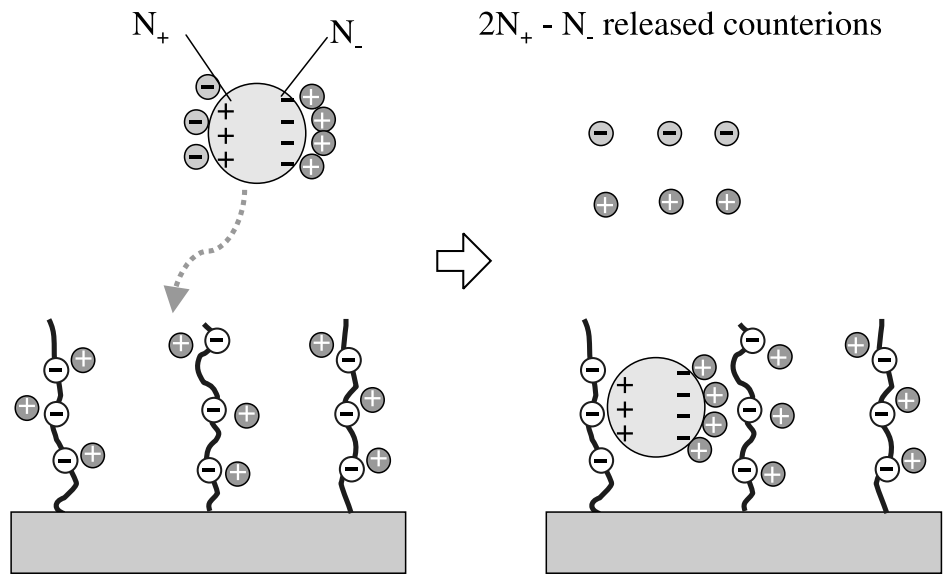

Fig. 5. Enumeration of the released counter- and coions during the process of protein adsorption. $N_{-}$is the number of negatively charged groups on the surface of the protein which is slightly greater than $N_{+}$, the number of positively charged groups on the surface $(\mathrm{pH}>\mathrm{pI})$. For each protein molecule a total of $\Delta N=2 N_{+}-N_{-}>0$ counterions of the protein and the brush layer is released. The decrease of osmotic pressure within the brush layer thus obtained leads to the strong adsorption of proteins at low ionic strength. At high salt concentration $c_{\mathrm{a}}$ the effect must vanish because the salt concentration in the brush layer is the same as outside in this case. See text for further explanation.

positive or negative charges. Recently, these patches have been discussed as the origin for the interaction of proteins with linear polyelectrolytes in solution [42-46].

We consider the uptake of a protein from solution (see Fig. 5) and enumerate the net release of counterions in this process. Because of $\mathrm{pH}>\mathrm{pI}$, the number $N_{-}$of negatively charged group on its surface is slightly greater than $N_{+}$, the number of positively charged groups on the surface. Evidently, these charges must be balanced by an equally high number of counterions of the opposite sign. Therefore each protein carries along $N_{-}$positive and $N_{+}$negative counterions. We now consider the immersion of the protein in the brush layer (see Fig. 5). The $N_{+}$positive charges on the surface of the protein now become counterions of the negatively charged polyelectrolyte chains. As consequence, $N_{+}$counterions previously immobilized within the brush layer as well as the $N_{+}$negative counterions formerly carried along by the protein in solution are released. On the other hand, the $N_{-}$negative charges on the surface of the protein carry along their $N_{-}$positive counterions which will increase the number of small ions within the brush layer again. The balance between the release and the uptake, however, is positive since a total of $\Delta N=2 N_{+}-N_{-}>0$ counterions have been released in this process. The concomitant lowering of the osmotic pressure within the brush layer is of the order of $k T \Delta N / V_{\text {brush }}$, where 
$\left.V_{\text {brush }}=(4 \pi) / 3\right)\left[(R+L)^{3}-R^{3}\right]$ is the volume of the brush layer on the surface of the particles.

A point to be made in conjunction with Fig. 5 is the size of the patches on the surface. These patches must necessarily be of a minimum size to ensure the strong correlation of the respective counterions. A single charge on the surface would not localize the counterion and there would be no difference upon putting the protein into the brush layer. However, patches of a few charges will bind their counterions more strongly and become multivalent counterions in turn when the protein is located within the brush layer. Therefore the counterion release mechanism is bound to the presence of charged patches on the surface of the proteins. The discussion of the patches on the surface of BSA by Seyrek et al. [44] point to at least one such domain on the surface of BSA which should be of sufficient magnitude.

Evidently, the above result that $\Delta N=2 N_{+}-N_{-}>0$ counterions will be released is a rather conservative estimate of this number inasmuch it assumes that the counterions of the negative patches (see Fig. 5) are carried along by the protein and introduced into the brush layer. If the negative patches are small or consist of single charges only, the respective counterions will not be carried along and $\Delta N$ will be concomitantly higher.

The counterion release mechanism now sheds light on the notion of irreversibility in the PMPA: Once the protein is adsorbed at a given salt concentration $c_{\mathrm{a}}$, the released counterions are removed from the system as depicted in Fig. 5. The proteins are thus firmly bound to the brush layer and can only be released upon raising $c_{\mathrm{a}}$ to higher values, that is, by introducing the necessary number of co- and counterions again. In this case most of the bound protein can be released again (see Fig. 4).

\section{Conclusion}

The driving forces leading to the strong adsorption of proteins in aqueous solution onto spherical polyelectrolyte brushes (SPB) has been discussed. In particular, it has been shown that raising the salt concentration $c_{\mathrm{a}}$ in the system leads to a controlled release of the protein. All findings can be discussed in terms of the counterion release force: Let $N_{-}$be the number of negatively charged groups on the surface of the protein which is slightly greater than $N_{+}$, the number of positively charged groups on the surface $(\mathrm{pH}>\mathrm{pI})$. For each protein molecule a total of $\Delta N=2 N_{+}-N_{-}>0$ counterions of the protein and the brush layer is released. However, at high salt concentration $c_{\mathrm{a}}$ the effect must vanish because the ionic strength inside the brush layer is the same as outside in this case. It is hence clear that the counterion release must be taken into account when discussing the adsorption of proteins onto polyelectrolyte brushes. 


\section{Acknowledgement}

The authors gratefully acknowledge financial support by the Deutsche Forschungsgemeinschaft, SFB 481, Bayreuth, and by the Fonds der Chemischen Industrie.

\section{References}

1. A. Baskin and W. Norde, Physical Chemistry of Biological Interfaces. Marcel Dekker, New York (2000).

2. C. Czeslik, Z. Phys. Chem. 19 (2004) 1861.

3. H. Kawaguchi, Progr. Polym. Sci. 218 (2000) 1171.

4. T. A. Horbett and J. L. Brash (Eds.), Proteins at Interfaces: Fundamentals and Applications, ACS Symposium Series 602. American Chemical Society, Washington DC (1995).

5. G. M. Fleer, M. A. Cohen Stuart, J. M. H. M. Scheutjens, T. Cosgrove, and B. Vincent, Polymers at Interfaces, Chapman and Hall, London (1993).

6. J. H. Lee, H. B. Lee, and J. H. Andrade, Progr. Polym. Sci. 20 (1995) 1043.

7. M. Zolk, F. Eisert, J. Pipper, S. Herrwerth, W. Eck, M. Buck, and M. Grunze, Langmuir 16 (2000) 5849.

8. E. Ostuni, B. A. Grzybowki, M. Mrksich, C. S. Roberts, and G. M. Whitesides, Langmuir 19 (2002) 1861.

9. A. Wittemann, B. Haupt, and M. Ballauff, Phys. Chem. Chem. Phys. 5 (2003) 1671.

10. G. Jackler, A. Wittemann, M. Ballauff, and C. Czeslik, Spectroscopy 18 (2004) 1671.

11. X. Guo, A. Weiss, and M. Ballauff, Macromolecules 32 (1999) 6043.

12. X. Guo and M. Ballauff, Langmuir 16 (2000) 8719.

13. X. Guo and M. Ballauff, Phys. Rev. E 64 (2001) 051406.

14. R. Advincula, W. J. Brittain, K. C. Caster, and J. Rühe, Polymer Brushes. WileyVCH, New York, Weinheim (2004).

15. P. Pincus, Macromolecules 24 (1991) 2912.

16. O. V. Borisov, T. M. Birshtein, and E. B. Zhulina, J. Phys. II (France) 1 (1991) 521.

17. A. Wittemann and M. Ballauff, Anal. Chem. 76 (2004) 2813.

18. T. Neumann, B. Haupt, and M. Ballauff, Macromol. Biosci. 4 (2004) 13.

19. A. Wittemann and M. Ballauff, Macromol. Biosci. 5 (2005) 13.

20. B. Haupt, T. Neumann, A. Wittemann, and M. Ballauff, Biomacromolecules 6 (2005) 948.

21. S. Rosenfeldt, A. Wittemann, M. Ballauff, E. Breininger, J. Bolze, and N. Dingenouts, Phys. Rev. E 70 (2004) 061403.

22. A. Wittemann, M. Drechsler, Y. Talmon, M. Ballauff, J. Am. Chem. Soc. 127 (2005) 9688.

23. C. Anikin, C. Röcker, A. Wittemann, J. Wiedenmann, M. Ballauff, and G. U. Nienhaus, J. Phys. Chem. B 109 (2005) 5418.

24. C. Czeslik, G. Jackler, T. Hazlett, E. Gratton, R. Steitz, A. Wittemann, and M. Ballauff, Phys. Chem. Chem. Phys. 6 (2004) 5557.

25. C. Czeslik, G. Jackler, R. Steitz, and H.-H. von Grünberg, J. Phys. Chem. B 108 (2004) 13395.

26. M. T. Record, C. F. Anderson, and T. M. Lohman, Quart. Rev. Biophys. 11 (1978) 103.

27. P. Sens and J.-F. Joanny, Phys. Rev. Lett. 84 (2000) 4862.

28. C. Fleck and H. H. von Grünberg, Phys. Rev. E 63 (2002) 061804. 
29. A. A. Meier-Koll, C. C. Fleck, and H. H. von Grünberg, J. Phys.: Condens. Matter 16 (2004) 6041.

30. C. F. Wertz and M. M. Santore, Langmuir 18 (2002) 1190, and further references given there.

31. G. Jackler, R. Steitz, and C. Czeslik, Langmuir 18 (2002) 6565.

32. R. Hariharan, C. Biver, and W. B. Russel, Macromolecules 31 (1998) 7514.

33. C. Biver, R. Hariharan, J. Mays, and W. B. Russel, Macromolecules 30 (1997) 1787.

34. R. Hariharan, C. Biver, J. Mays, and W. B. Russel, Macromolecules 31 (1998) 7506.

35. Y. Mei and M. Ballauff, Eur. Phys. J. E 16 (2005) 341.

36. G. Manning, Ann. Rev. Phys. Chem. 23 (1972) 117; and further references cited therein.

37. see the discussion of this point in M. Deserno, C. Holm, J. Blaul, M. Ballauff, and M. Rehahn, Eur. Phys. J. E 5 (2001) 97.

38. P. M. Biesheuvel and A. Wittemann, J. Phys. Chem. B 109 (2005) 4209.

39. H. Ahrens, S. Förster, and C. A. Helm, Phys. Rev. Lett. 81 (1998) 4172.

40. B. Das, X. Guo, and M. Ballauff, Progr. Colloid Polym. Sci. 121 (2002) 34.

41. A. Jusufi, C. N. Likos, and M. Ballauff, Colloid Polym. Sci. 282 (2004) 919.

42. T. Hattori, R. Hallberg, and P. L. Dubin, Langmuir 16 (2000) 9738.

43. K. R. Grymonpre, B. A. Staggemeier, P. L. Dubin, and K. W. Mattison, Biomacromolecules 2 (2001) 422.

44. E. Seyrek, P. L. Dubin, Ch. Tribet, and E. A. Gamble, Biomacromolecules 4 (2003) 273.

45. R. de Vries, F. Weinbreck, and C. G. de Kruif, J. Chem. Phys. 118 (2003) 4649.

46. R. de Vries, J. Chem. Phys. 120 (2004) 3475. 